\title{
COMENTARIOS
}

\section{ECOECONOMIA Y DESARROLLO SOSTENIBLE COMO CONCEPTOS CENTRALES PARA UNA PROPUESTA SERIA AL PERÚ}

\section{ECOECONOMY AND SUSTAINABLE DEVELOPIMENT AS PRINCIPAL CONCEPTS OF A SERIOUS PROPOSAL FOR PERÚ.}

\author{
Jesús H. Córdova Santa Gadea ${ }^{12,3}$ y Sonia E. Moquillaza Villar ${ }^{2}$
}

\author{
"Silo la Pra- Social posibilitara el Desurollo. \\ pero sólo si este es sostenible aquellu perdurari \\ El rewo del momonto actual es hacer que ambus \\ rayan de la mano para hacer del peria un \\ moveclo viable en el riempo."
}

\section{RESUMEN}

La contundencia de los hechos ha mostrado una verdad indiscutible: la vida en la Tierra se sustenta en una cantidad limitada de energia que producen organismos como las plantas verdes. Resulta por ello más urgente que nunca poner en práctica medidas que aseguren el mantenimiento — cuando menos - de tal cantidad de energía. La creciente tasa de deforestación y contaminación (polución), como producto de la "modernidad", permite avizorar un futuro de la humanidad seriamente comprometido y no es necesario siquiera esperar a la generacion de nuestros hijos para apreciar sus negativos efectos. A inicios del siglo XXI ya los vivimos e inclusive los podemos cuantificar. Habrá que decidir que hacer para evitar uno de los mayores desastres ecológicos: la asfixia del planeta. Las unicas fórmulas capaces de evitar este desenlace se encontrarian dentro de lo que involucra el concepto de Ecodesarrollo o Cesarrollo Sostenible, inspirador de la forma más revolucionaria de filosofia de Estado, la Ecoeconomia. Aqui se ofrecen algunos preceptos fundamentales en los que se sustentan y se hacen notar los peligros de no actuar rápidamente según su consejo en paises que como el Perú poseen una enorme magnitud de diversidad biológica (ocupa uno de los primeros lugares entre diez que son mundialmente reconocidos). Por esta caracteristica nuestro pais es considerado megadiverso. Para propósitos de Desarrollo Sostenible, la condición de megadiverso en un mundo donde existe una globalización del mercado se considera una enorme fortaleza, capaz de generar - si existe decisión politica- una fuenle inagotable de poiencialidades y oportunidades, que permitirá al pais alcanzar significativos y crecientes niveles de bienestar en el corto, mediano y largo plazo.

Palabras clave: Desarrollo sustentable, Biodiversidad, Eco-economia, Calidad de vida, Perú

\begin{abstract}
The forcefulness of the facts has shown an unquestionable truth: the life on Earth is sustained by a limited quantity of energy that is produced by organisms like the green plants. It is urgent to put into practice measures that assure the maintenance of at least such a quantity of energy. The growing deforestation rate and contamination (pollution), as by-products of the "modernity", allows us to watch the seriously committed humanity's future, not being necessary to wait to the generation of our children to appreciate their negative effects. In the beginning of the XXI century we already live them and inclusive we can even quantify them. It will be necessary to decide what to make in order to avoid one of the biggest ecological disasters: the asphyxia of the planet. The only formulas that can to avoid this outcome lie within the concept of Eco-Development or Sustainable Development, inspiring in the most
\end{abstract}

1. Museo de Historia Natural-Instituto de Ciencias Biológicas "Antonio Raimondi". Facultad de Ciencias BiológicasUNMSM integrante de la Comisión Nacional de Diversidad Biológica (CONADIB)

3.Correspondencia a J.H. Córdova S. G. , correo electrónico: d190072@unmsrn edu.pe 
revolutionary ideas in State philosophy-Eco-Economy. Here we offer some fundainental precepts on which Ecoeconomy is based, and we point out the dangers of not acting quickly enough according to their advice in countries that possess an enormous magnitude of bioloyical diversity as the Feru (which is one of the top ten recognized worldwide). For Sustainable Development purposes, being megadiverse condition in a world of globalized markets amounts to an enormous strength, that can generate — provided there is a political will-- an inexhaustible source of potentialities and opportunities, which could enable the country to reach significant and growing levels of benefits in the short, medium and long term.

Key Words: Sustainable Development, Biodiversity, Eco-Economy, Life-Quality. Peru.

\section{INTRODUCCIÓN}

la Conferencia de las Naciones Unidas subre el Medio Ambiente y el Desarrollo, Ilevada a cabo del 3 al 14 de junio de 1992, permitió comprobar el crítico momento en que estuvo y aún continúa estando el planęta Tierra como producto de la intensa actividad humana.

La sobreexplotación de los recursos y la contaminación ambiental están produciendo daños a escala mundial. La creciente tasa de deforestación y extinción de especies, así como el aumento del bióxido de carbono y el debilitamiento de la capa de ozono en la atmósfera cstán propiciando cambios climáticos globales tales como el sobrecalentamiento y el crecimiento de la penelración de radiación exógena. Algunus de sus efcctos ya se dejan sentir en diferentes puntos del planela en la forma de inundaciones y sequías prolongadas, lo que permite avizorar un futuro global próximo definitivamente comprometido, de no mediar radicales modificadores del comportamiento humano.

En la mencionada Conferencia se suscribió, entre otros, documentos tales como la Carta de la Tierra, en la que se enuncian los principios fundamentiles que dehcrán rugi: la conducta económica y ecológica de li., naciones y los pueblos; la Agenda 21 , que viene a ser un programa de acción presente y futuro para las principales áreas que involucran la relación ambiente-economía; así como los Convenios, entre los que destacan los referentes al Cambio Global del Clima, al Mante- nimiento de la Biodiversidad (CBD) y el referente a la Gestión Forestal.

El Perú, presente en la cita cumbre. suscribió esos documentos en un acto csperado dentro de los círculos científicos nacionales si se tiene en cuenta que se reconocen en el mundo a solo diez países que posen megabiodiversidad, entre los que se encuentra el nuestro y como uno de los primeros. El reto que representa para cl país, poseer y conserval lal biodiversidad lo obliga a contar con una estratugia que permita colocar sus ingentes recursos naturales, en disposición de ser utilizados de manera sostenida por las actuales y futuras generaciones de peruanos y, por extensión. en beneficio de las de la humanidad. Es imprescindible tener muy clara esta idca, tanto a nivel de gobierno como de cierto sector de científicos que sienten temor ante el movimiento conservacionista (con base cientifica y positivo), que es cualitativa y cuantititivamente distinto del superado "proteccionista" (instintivo, negativo y hasta prostituido), pues aquél por definición no puede ser un freno a ninguna aspiración legítima de crecimiento individual. Considera síque para que éste sea trascendente, debe efectuarse dentro de un marco de referencia de responsabilidad, solidaridad y consideración con el resto del mundo, esto es, con la multiplicidad de seres que lo habitan.

Los peruanos podemos ubicarnos en el sentido positivo de la Historia, si de una buena vez incorporamos a nuestra política gubemamental y a nuestro diario accionar las recomendaciones y planteamientos de esa nueva 
concepcion diel mundo que es el Ecodesarrollo. Por sute se entiende al proceso que jermite alcanzar el desarrollo económico dr pueblos y naciones, en carmunia cun su umbiente. En csencia, más que un conjunto de acciones científico-politicas. debe ser idealmente producto de una ecosofía una filosofía de vida en la que el respeto al entorno puede estar presente hasta en la más elemental de las acciones lumanas (Ame, 1981: Arhem. 1990).

En las actuales circunstancias su aplicación alcanza ribetes singulares, pues de no ceñirse a sus preceptos no podremos — según lo estiman algunos técnicos radicalesevitar nuestra propia destrucción y dejarlo para otra ocasión; sería quizá den laciado tarde. Para los más mo arados y prácticos, significa perder una de las últimas potencialidades-oporlunidudes--si no la última-de poder mejorar en favor de paises como el nuestro, las actuales relaciones de cambio que se tienen con las grandes potencias del Hemisferio Norte, cuyos programas de asistencia y planes de desarrollo no se rigen en su mayor parte por el principio del desarrollo sostenible siendo por ello discontinuos e ineficientes. Auncue en la actualidad existen organismos tales como el Programa de las Naciones Unidas para el Medio ambiente (PNUMA), el Banco Mundial y la Agencia Interamericana para el Desarrollo (AID) para otorgar apoyo económico, son cada vez más exigentes respecto a la existencia de clara:, definidas y favorables decisiones políticas conservacionistas-ambientalistas en los gobiernos solicitantes.

Sea como fuere, parce imperativo y urgente no mantener el actual estado de cosas, y debe tenerse una visión a futuro del Perí y hacer el esfuerzo de dar a nuestro país la oportunidad de encomrar un camino que le permita alcanzar finalmente el biencistar al que por suriquisima historia tiene legítimo derecho y cuya Constitución Política, además, propugna.

\section{Ideas fuerza}

El proceso de aplicación del Ecodesarrollo se sosticne en cuatro pilares fundamentales: la Economica, la Conservación, el Derecho y la Educación. Estos clementos deben actuar concurrentcmente. : i alguno fallase, no debe esperarse ecodesarrollo. Un proceso que complementa a los cuatru pilares, es el de la descentralización vía una regionalización concriterio cientifico.

La Economía us básica, por cuanto debe ser la encargada de hallar capitales, suministrar los recursos económico-financieros que denanda «implemontar» el ecodesarrollo. así como su correcta administración. También debe ser ol irea encargada de determinar la relación costo-bencficio de las accciones conservacionistas. Por tales atributus, en su conjunto bien puede ser calificáda como una moderna disciplina ad-hoc: la Licoeconomía.

La ciencia de la Conservacion y Murrejo de Recursos Naturales renovables - fase final o aplicada de los estudios sobre biodiversidad - nos provee de propuestas cientificas para el mantenimicnto y uso sosicnible de los mismos, en tanto que el Derecho nos oturga el marco lernt o legislaciún ambienlal dentro del cual lus accion _ 3 de conservación, manejo y uso de recursos se llevan a cabo. Es la parte que permite exponer las "reglas del juego", entre otros. para la inversión nacional y/o extranjera en materia de recursos naturales. Importante será que tal legislación sea promotora.

La Educación, si está sintonizada con la modernidad, resultará esencial en cl cambio en la manera de pensai de la sociedad peruana $y$, en consecuencia, en el de sus futuros gobernantes. Necesariamente la educación a nivel inicial, primario, secundario y superior debe acompañar a las acciones conservacionistas, justamente para que éstas se entiendan cada vez mejor en cada nueva generación de peruanos. La sociedad debe estar persuadida que el desarrollo sostenible es la mejor altemati- 
va para alcanzar el bienestar y finalmente, la puz social. A la vez, se precisa de un margen sustancial de paz para que tal clesarrollo pueda despegar. Asi, paz y desarrollo sostenible aparecen como elementos virtualmente indisolubles.

El proceso de descentralización a través de una regionalización es, sin duda, muy complejo para una realidad como el Hurí. Aquí existe un conflicto entre las delimitaciones artificiales de las regiones formuladas hasta la fecha (por criterios políticos) y las naturales. Dos opciones destacan entre éstas últimas: el de las distribuciones geográficas emolingüisticas y las que se establezcan por biorregiones. Serios indicadores señalan que una regionalisación natural es la que pasaría por la línea de menor resistencia en su aplicación y la que brindaría los mayores logros en el menor lapso.

\section{Conceptos básicos}

Un ecosistema es definido como una unidad funcional básica de la naturaleza, que comprende a un grupo de organismos vivos (componentes bióticos) y al ambiente físico y químico (componentes abióticos) donde el los viven. Según los especialistas modernos, la Ecologia y la Economia tienen grandes semejanzas, puesto que ambas sc ocupan de la dinámica de productos valiosos que circulan en una compleja trama de productores y consumidores. Así como la Economía funciona en base al dinero, un ecosistema lo hace en base a la energia, toda ella proveniente en principio del Sol. Un ecosistema no cuenta con un "flujo de capital" energético ilimitado como podría imaginarse. sino que depende de un "presupuesto" relativamente reducido, debido a que los organismos vivos utilizan la energía solar indirectamente, teniendo acceso sólo a una peçueña fracción de ella. que es convertida en una forma de energía química almacenada (materia orgánica) por las plantas verdes, mediante el proceso conocido como folosintesis. Este "producto almacenado" (las plantas son los productores prima- rios) es utilizado en parte por las mismas plantas para su propio mantenimiento en tanto que la cantidad restante es aprovechada por los animales herbivoros (o red de consumidores primarios) que se alimentan de ellas. Asímismo, éstos son devorados por los animales carnivoros (o red de los comsunidores secundarios) dentro de los que está la especie humana. Cuando muere alguno de los anteriores. sus reslo:i son aprovechados por los organismos desintegradores (o red de los detritivoros a consumidor(s) terciarios). los cuates recirculan sus prośuctos como moléculas clementales (red de elementos orgánicos) que se encargan del reciclaje natural, pues parte do tales moléculas son transferidas al ambierte. cerrando así el ciclo de la trunsferencia energética. Pero otra de las cosas que se sabe bien, es que toda la materia orgánica que se asimila y que no es utilizada en los procesos de crecimiento individual es virtualmente "quemadia " por el proceso esencial de larespiración, que mantiene el metabolismo de plantas y animales, generando el calor necesario para que tal proceso se realice. Esta forma de energia (el calor) se pierde hacia el ambiente, siendo la razón principal por la que todo ecosistema necesila un flujo cuntimuo de energía proveniente del Sol $y$, por supuesto, de las plantas verdes (Gosz, et al., 1978; Ricklefts, 1998).

De lo expuesto no debe inferirse que el mensaje es no ingresur a o no utilizar ecosistemas recién descubiartos o no conocidos por algún grupo humano extraño a él. sino que si se decide hacerlo, debe ser iniciado por personal especializado a fin de no romper los clelicados procesos existentes en cllos. Si un ecosistema posee componentes biológicos de utilidad para la especie humana, lo ideal sería potenciarlos, así se los podrá tomar o cosechar sin temor ni peligro futuro alguno, pues se estaría extrayendo sólo excedentes de tal ecosistema. Si no se pudieran potenciar, se los podría extraer racionalmente, es decir, en cantidades tales que no comprometan la supervivencia de especie alguna. 
Ecoeconomia: inevitable y moderna disciplina para el diseño de una eficiente politica de gobierno

"Algun día en lo más allo de las monrañas se lan-ari al wiento la palabra ;Economia! y el eco respondera' Ecologia!"

(Richard Webb. 1993)

"El optimismo de la reactivación económica del Perif reposa en sus recursos naturales. Es un pais privilegiado. Los recursos naturales renovables tiemen valor estrategico."

(Richard H'ebb, 2001)

Todo individuo o agrupación social que aspira a ser trascendente, debe estar inspirada en una vision a futuro de lo que considera debe ser aquella realidad en la cual se alcanza el bienestar de sus semejantes. Para ello se debe partir de la premisa de que el Perú existe como una entidad afectada por la globalización, es decir, que está influenciada por sus relaciones con el resto de entidades semejantes del globo terrestre y en donde los países no pueclen scr considerados meras islas capaces de alcanzar cada uno de ellos de manera independiente su "propio" bienestar o desarrollo sin considerar el de los restantes.

Hechos tales como el sobrecalentamiento y el agujero de la capa de ozono de la Tierra - causados por la actividad humana irresponsable a gran escala - o eventos naturales tales como el Fenómeno de "El Niño" nos han hecho comprender que lo que ocurre de perjudicial en el ambiente de un determinado lugar del planeta, repercutc la mayor parte de las veces en otros de alguna otra manera, también perjudicial y a veces con mayor intensidad. Tales aconiecimientos han llevado al razonable convencimiento de que existen nexos y "algo común" que se comparte con todos los países del mundo. Ese "algo" es el ambiente o entorno.

Una consecuencia del concepto de globalización es que ha quedado establecido que los humanos somos una fracción de una enormidad de habitantes que comparten un mismo "hogar" (el ambiente o entorno) y que no podemos quedamos con todo lo que quisié- ramos tomar de él, porqué con alta probabilidad nos estaríamos quedando con la "porción" perteneciente a algún otro de tales habitantes. Casi sin esfuerzo se puede intuir la cxistencia un "presupuesto natural" en el mundo, clel cual una región o un pais tendría una "parte" que debe conocer y administrar apropıadamente, para no poner cil riesgo su supervivencia y la de los demás en el tiémpo.

Antes se mencionó que la Ecología tiene muchas similitudes con la Economia a nivel global, de pais, de región o de manera más precisa, de ecosistema (porción del universo natural razonablemente susceptible de delimitación, medida, experimentación y manejo [Odum. 1972; Margaleff, 1974; YCEA, 1994; Fernández et al., 1998]). Vimos que ambas disciplinas se rigen por presupuestos. Para el ecosistema la energía es la unidad de transacción. Para la Economía lo ss el dinero. El presupuesto del ecosistema puede wiminarse por la abundancia de los organismos productores. Si hablamos de un bosque, el presupuesto básicamente lo establecerá la magnitud de su superficie foliar (o de las hojas verdes), pues ella determinará la canticlad de luz que podrá convertir en cantidad de energía de utilidad biológica (Goszet al., 1978). Esta última cantidad es el "presupuesto" con el que contará dicho ecosistema para mantener indefinidamente a todos los organismos que de manera natural en él se encuentren, incluida si fuere el caso la especie humana.

El problema reside en que esta última históricamente se ha comportado de forma altamente invasora, y por ello opertunista y egoísta, y ha tomado-especialmente cn los paises desarrollados - no sólo mucho más de lo que le correspondía de un ecosistema hasta agotarlo, sino que además lo ha ensuciado de tal manera con los desechos o productos de su intensa actividad "desarrollista-consumista", que ha logrado que muchos ecosistemas sean ya incapaces de recuperarse. Unos por explotación directa, otros por "contagio". Detectada esta situación, los grupos humanos o bien 
se desplazaron hacia otros ecosistemas para hacer lo mismo de tal suerte que ahora han ocupado virtualmente toda la faz de la Tierra, o bien los que no emigraron, se quedaron en un medio cada vez más contaminado y menos productivo, dándose cuenta de que "misteriosamente" su economía y posibilidades de desarrollo también estaban cada vez más amenazadas.

Es aquí donde se encuentran la Ecología y la Economía, pues ésta ha terminado por admitir que uno de los elementos más poderosos que deben considerar las sociedades humanas en la formulación de sus estrategias y presupulestos de Estado es el componente ambiental, específicamente lo que los economistas califican también como su entorno. Virtualmente no hay economista moderno que no ubique al entorno natural como el elemento más inclusivo o de jerarquia mayor, dentro del marco en el cual deben manejar sus polílicas para diseñar y controlar sus indicadores macrocconómicos (Cabrera, 1999). Las uniclicle: sujeto (it la actividad de los economistas estadistas son los países (u otra similar delimitación política), que no son otra cosa que porciones arlificiales de un universo natural. Fsitc hecho no parece tener solución y de aquí nacen las terribles contradicciones actuales en la forma de innumerables y complejos problemas "de Estado" que la Economía tiene que afrontar. Una opción más "económica" es, entonces, dirigirse hacia una Economía cada vez más "natural" (una vez. más por la linea de menor resistencia) que permita hacer la mejor gestión de cada "porción artificial" ("naturalizar" lo artificial).

La forma de ejecutar nuestras acciones, actuales y futuras, es que ellas deben ser hechas con un sentido de respeto al ambiente o entorno natural que nos rodea y al cual los gobernantes deben conocer, cuando menos, en sus aspectos más relevantes (los científicos y los técnicos idealmente hasta el detalle). Tal disposición nos introduce hacia una inevitable serie de conceptos que, por tener esa dimen- sion global, son calificados como "tendencias mundiales" o megatendcncias. También es posible cicontrarla en expresiones yue tienen un sentido comparativo, como el singular atributo que posee el Perú en materia de la magnitud de su diversidad biológica frente a la inmensa mayoría de los demás paises del mundo: él es considerado megadiverso, que no es otra cosa que el reconocimiento cientílicamente comprobado de que en la actualidad somos uno de los diez paises del orbe que poseen la mayor variedad y variabilidad de formas vivientes por unidad de superficie, y que junto con Brasil, Colombia, Ecuador y Venezuela rcúnen alrededor del $70 \%$ de la superficie terrestre considerada como el "pulmón" que manliene con vida al planeta. Esto signilica que estos países ticnen mayores ventajas comparativas en este tema respecto a los demás.

¿Qué connotaciones tienen para el diseño de las políticas de Estado las megatendencias en un país megadiverso como el Perú?. Una de las principales es que como país que ha suscrito normas juridicas de carácter internacional o global y ademas vinculantes, tales como el Convenio sobre Diversidad Biológica (CBD) y el de Cambio Climático. Pertenece a aquel grupo de naciones privilegiadas hacia las cualcs ciertas megaiendencias van a dirigirse con mayor intensidad, pero que debe significar para nosotros fuente de poiencialidades y las mejores oportunidades para alcanzar un desarrollo basado en nuestros recursos naturales renovables y los recursos humanos nacionales especializados en estos, además de contriburi a la sobrevivencia del mundo. Los paises del Hemisferio Norte, que ya agotaron o están a punto de agotar su biodiversidad y contribuyeron con la mayor parte de la polución (contaminación de origen humano) global existente, se encuentran dispuestos - por múltiples razones - a invertir para que paises como el Perú, por ejemplo, utilicen racionalmente sus recursos renovables. Fn otras palabras, tienen evidentes y urgentes niveles de demanda de productos $y$ servicios de nuestra diversidad biológica, en 
Limto que nosotros disponemos de una enorme oferla. la cual debemos administrar con rspunsabilidad y esto en el entender moderno. supone hacerlo respetando al enlorno ( $\mathrm{sin}$ depredar ni contaminar).

Ante este licho, adoptar un estilo de hacer politica gubernamental convencional o tradicional resulta no solo obsoleto sino definitivamente peligroso y hasta suicida. La política por adoptar necesariamente dube estar inserta dentro de esa nueva megatendencia, la Ecoeconomia, cuya base o teoría bien puede ser calificada como una Ecosofia y su praxis la Economia ligada al descrrollo sostenible.

Por Ecosofia hay que entender aquella comiente filosófica que propone que todos los actos de la actividad humana se encuentren inspirados en un sentimiento de absoluto respeto por los elementos del entorno, tales como el uso racional de los recursos naturales renovables y la no contaminación del ambiente que los rodea. Es una filosofía de la naturaleza investida de valor :ormativo (Arne, 198I; Arhem, 1990).

Li clesarrollo sostenible es más bien el pruceso pur el cual se alcanza el desarrollo económico de los pueblos sin deteriorar su ambiente ni extinguir sus recursos naturales renovables (Prescott ct al., 2000). Tal procedimiento idealmente deberá privilegiar cl uso de los recursos renovables en desmedro de los no renovables, tanto como sea posible, reconociéndose que la necesaria explotación de los segundos es aún la mejor manera de tener la "liquidez" necesaria para alcanzar los objetivos de emergencia extrema, que por su naturaleza pertenecen a los de muy corto plazo. Por otro lado, como procedimiento el desarroIlo sostenible no es compatible con el concepto de democracia dirigida "de atriba abajo" (impuesta por la cúpula gubernamental haçia su pueblo), sino "de abajo hacia arriba" mediante el concepto moderno de democracia ampliamente parficipativa y curcertadora, que debe incluir a los especialistas académi$\mathrm{cos}$, asi como a las comunidades nativas y cam- pesinas, en estrategias de tipo regional dada la enorme complejidad de nuestro territorio. Se debe superar entre otros el concepto y $s / a-$ lus de pais exportador de simplemente materias primas. Debemos dar a éstas el máximo del valor agregado previo a sus exportaciones, pues sólo así la tasa de recuperación de lo invertido podrá ser significativa e intercsante; de otro modo, contribuirán a acortar el margen de gasto entre el producto expurtado y su retorno del cxterior en forma acabada.

\section{Perú: Estrategia Nacional sobre Diversidad Biológica - Reflexiones}

En esta parte se comenta un conjunto de hechos que evidencian que aún estanos soportando un considerable lisire que impide el ingreso del país a la senda del desarrollo sostenible. Recientemente se publicú el doctimento "Perí: Estrategia Nacional sobre Diversidad Biológica (ENDBP)", que fue promovido $y$ editado por el Consejo Nacional de Ambiente (CONAM). El primer autor (JHC), como integrante de la CONADIB, tenía la csperama de que este documento coniagrara propuestas audaces y trascendentales que originalmente contenia, pero en la redacción final prevalecieron al gunas posiciones conservadoras, y así se ve que la versión publicada difiere notablemente de su último borrador en aspectos centrales. Aunque como toda Estrategia Nacional, no debería ser un "corsé" o una "camisa de fuerza" para el pais, sino más bien disponer de veloces mecanismos para perfeccionarse, parece que tendrá los "atributos" de los primeros, al menos por algún tiempo. Veamos por qué.

En primer lugar, ha variado sustancialmente la visión a futuro paru el Perí. El último borrador a este respecto decía que al 2015: "Se aprovechan las oportunidades que ofrece la enorme Diversidad Biologica del P'erú para su desarrollo económico, social, tecnológico y ambiental, teniendo como base la utilización sostenible de sus compo- 
nentes. Se proyectan como Unidades de Planificación Sosten ible y de Mimejo, las Bio-Regiones autónomas, de:icentralizadas, democrálicis y articuladas, a fin de robustecer una esiruciura exportadora agroindustrial, pesquera y ecoturística". Lo que se publicó dice: "Al 202 l el Perí es el pais en el mundo que obtiene para su población los mayores beneficios de su Diversidad Biológica conservando y usando sosteniblementc, y restaurando sus componentes para la satisfacción de sus necesiclides básicas, el bienestar y la generación de riquc a para las actuales y futuras generaciones". El texto de la ENLBPP se "siente" laxo (retorico) y nada comprometido. y se aleja en aspectos de fondo del citado borrador.

Ina primera concesión que se desprende del texto aprobado, es haber retrasado en más de un quinquinio (del 2015 al 2021) el alcanzar el desarrollo sostenible y el bienestar de los peruanos, justo cuarudo los plazos de las metas a corto, mediano y laryo plazo se han reducido a la mitad, dado que virtualmente existe una revolución gluhal cada dos años. La segunda concesión c:i 110 mencionar en absuluto lo imperativo de la descentralización del Perú al menos pos biorregiones, como un derrotero para orientar la Economía Nacional y sus prioridades.

Fucra de la "visión", se aprecia algo que puede ser fuente de futuros problemas y tiene que ver con un concepto esencial de un tema emergente: el cle especie exólica. No se entiende como se ha podido aceptar la afirmación que figura en la pág. 104 (Glosario ENDBP), que dice: "el término cxótico sc utiliza para los organismos que no son originarios del pais". Hay que recordar aquí el texto del Informe Nacional del Perú presentado en Brasil en la Reunión de Trabajo sobre Especies Exóticas Invasoras (Del Rín et al., 2001, citado por Ojasti, 2001) a nivel de la Comunidad Andina de Naciones (CAN). Tal texto dice a la letra:"Es pertinente hacer algunas reflexiones alrededor de cste punto, como profilaxis académica (teórica) y legal (práctica). El uso del término exólico en el Perú no significa lo mismo para instituciones que tiencu que ver con la probiemática fito y zoosanitaria, como es el caso del Instituto Nacional de Sanidad Agraria (SENASA) que es entendido como orizmdo o procedente de otro pcrís, y se estaria aplicando sólo para aquellas especies en lasi que la especie humana es su 'vector' intencional o involuntario. Otras institucioncs mús vinculadas al Conven io sobre Diver idad Biológica, consideran que un concepo di especie exórica debe ser más amplio, adoptándose aquel que precisa que debe recibir tal denominación toda "especie que se hal la fuera de su área de distribución natural" (UICN, 2000). Estc concepto, prescinde de los limites politicos que tienen las naciones y aparece como el más aparente. De cualquic modo, es necesario llegar a tener un ímico y sulisfactorio concepto de especie exótica, a fin de evitar peligrosas confusiones futuras". El párrafo inmediato dice: "Surge la pregunta aqui si en casos de determinados lénómenos naturales. como avalanchas, inundaciones, huracanes. o como se indica desde el punto de vista del SENASA. que existen especies exóricas que se diseminan en forma natural, como son hongos, virus y bacterias a través de vectores como insectos, nemátodos y aqucllos insectos que tienen publaciones migrantes como las langostas, existen suficientes evidencias de que innumerables especímenes no nativos son transportados desde lugares lejanos, o simplemente desde ciertos hábitats, hacia otros donde antes no existían. convirtiéndose en 'colonizadores' o 'fundadores' de nuevas poblaciones y desde nuestro punto de visia, maturales. En cualquier caso parecería "iner el derecho (y hasta la necesidad) (le ser denominados también exóticos pero ite hábilat. Para el SENASA lo que tenemos como nálivo dentro de un país simplemente no porlemos considerarlo exótico." Juhani Ojast (2001). experio internacional en el tema, ha aceptado la definición de especies exóticas que dio el Artículo 8 h de la CBD que se ajusta a lo expresado en la concepción precedente, y significa que 
nuestra estrategia debe, al más breve plazo. recrificur su definición y optar por la propuesta de la CBD-CAN, y lo argumentado en nuestro Informe Nacional sobre Especics Exóticas Invasoras (Del Rio et al., 2001).

Ura siluación inconveniente tiene que ver con el carácter de "corsé" o "camisa de fuerza" que para el Perí tendrá la presente Estrategia. Dos de sus Principios Rectores asi lo sugieren pues dicen lo siguiente: "La Estrategia Nacional sobre Diversidad Biológica es el documento formal $y$ guía para la planificación y gestión de la Diversidad Biológica del país" y el que le sigue precisa: "Todas las estrategias relacionadas a la Diversidad Biológica tendrán como marco orientador la Estrategia Nacional sobre Diversidad Biológica". La lectura detenida de la Estrategia no señala cómo ésta se va a perfeccionar rápidamente. Se espera que los organismos competentes empiecen por este último inconveniente y se lo supere en el plazo más breve en función de los más elevados intereses nacionales.

Una Estrategia Nacional para la Diversidad Biológica y el Desarrollo Sostenible que hubiera recosido los aspectos inedulares de las Estrategias Biorregionales debió ser el instrumento o marco ideal dentro del cual se hubieran llovado a cabo todas las ramas trascendentes de la actividad humana, con la certeza de que la variable, salud del entorno estaria asegurada, y se convertiria, además, en un immejorable indicador de la decisión política como pais cle ir hacia el ecodesarrollo. No obstante, en el estado actual es posible que la Estrategia Nacional ubique al Perú en una posición favorable para acceder al apoyo de entes financieros internacionales que ayudan a los paises que exhiben una politicu cimbienlal sostenible y definida, pero que a la vez vienen siendo celosos vigilantes de los desvios que al respecto pudieran ocurrir. No se debe olvidar que una Estrategia ampliamente participativa, necesariamente la harán suya lodos los que parliciparon en su elaboración, pues sentirán que pasarán del grupo de los señaladores de obstáculos y problemas al de los formuladores y ejecutores de sus soluciones. Fsto significa en suma, que dentro del siguiente quinquenio, se deben sentar las más sólidas bases para alcanzar el ansiado desarrollo económico pero con respeto al ambiente y a las culturas nativas que se mantienen aún en nuestro Perú en significativa armonía o integración con su entorno y de las que se tiene aún mucho que aprender.

Se ha mencionado que el proceso de descentralización deberá rehacerse en el Perú. Más que una simple opción, es un imperativo para alcanzar un genuino Desarrollo Sosicnible. F.l mecanismo utilizado ha sidu y será a través de una regionalización que aparece nuy compleja por las características del Perú. Si ésta se intenta hacer tal como se him en el pasado, nuevamente se produciráu conflictos e impasses dentro y entre esas delimitaciones arificiales que reciben el actual nombre de regiones. Ellas fueron hechas basadas fundamentalmente en criterios políticos y aún puede decirse que algunas subsisten hasta la liecha. Conflictuan también con las liniones naturales cuyos criterios para definir límites todavía son materia de esiildio. En áreas como las de nuestra Selva, una hipótesis por explorar debe ser la delimitación en base al criterio de las distribuciones geogrificas etnolingüisilicus. Las primeras evaluaciones al respecto parecen más que satisfactorias. Para otras áreas de nuestro país, la opción alternativa puede ser una delimitación por biorregiones, para las cuales ya existen estrategias. Existen cinco y pertenecen a sendas Macro Regiones. Por razones que no entendemos, cinco también son los departamentos que no están incluidos en ninguna: Amazonas, Apurimac, Huánuco, lca y Pasco (ENDBP 2001).

Parece necesario exponer esta forma de entender al mundo y a los pueblos que lo conforman - entre los que está el nuestrodado que hasta el presente en el Perú, los políticos no han dado señales de conocer a cabalidad la Ecoeconomía. Y ésta es definiti- 
vamente una alternativa política novedosa, inédita para nuestra rcalidad, que por su carácter de emerger naturalmente, se aproxima mucho más que las políticas ya probadas y caducas, a lo que como país precisamos y aspiramos para nuestros hijos. Con seguridad esta disciplina hallará las soluciones que se requieran porque posee las herramientas necesarias. Por otro lado, se dice que es propio de los jóvenes intentar lo nuevo y el asumir riesgos. Si nos consideramos uno de ellos ya sea de cuerpo y/o de mente, no se debe reincidir "apostando" por propuestas trilladas cuyos fracasos ya se conocen.

Debe enfatizarse que esta visión de desarrollo para nuestro país sólo se alcanzará cuando exista la decisión politica gubernamental de que para aprovechar las oportunidades que brinda la Diversidad Bioiogica en el marco de la globalización de los mercados, de la información y de la problımática ambiental, se debe promover alianzas estratégicas entre el Estado y sectores privados comprometidos y proactivos.

No queremos concluir sin entregar una cita textual de Ráez-Luna (2001) por considerarla muy pertinente, actual y válida para lo que aquí hemos querido presentar: "el reto de gestión consiste en obtener un balance reflexivo e informado entre el principio de precaución y los derechos humanos. Para alcanzar dicho balance necesitamos una perspectiva integradora que articule economía, ecología y etnología, asumidas en sentido amplio pero frente a realidades concretas. Esta integración exige un esfuerzo intelectual consciente por parte de investigadores y administradores, un verdadero repensamiento de nuestras respectivas funciones. En particular, debemos entender la gestión como conjugación de 'lo social' y 'lo ecológico', abandonando la tendencia miope a reducir lo social a 'amenazas' y lo ecológico a 'impactos'. En buena cuenta, hablamos de balancear el derecho humano a la vida con la conservación de la naturaleza.". Más adelante agrega: "A una alianza sólida entre investigación y gestión no se llega por defecto, ni por edictos, ni por acumulación de doctorados: publicaciones; se llega a través de esfuerzos intelectuales conscintes y voluntades concertadas entre investigadores y administradores Este es un Ilamado al diálogo". Agregaríamos que el diálogo debería ser inmediato y las acciones tomarse en seguida, pues el inicio del proceso hacia el bienestar del P'erú no puede ni debe esperar más tiempo.

\section{BIBLIOGRAFIA}

Arne. N. 1981. Ekologi. Samhalie och Livestil. (Stockolm), $116 \mathrm{pp}$

Arhem. K. 1990. Ecosotia Makuna. Pp: 105-122. En: La Selva Hunanizada. (Correa. F. Cd.) ICAN-Fondo Editurial CEREC:(Colombia). $255 \mathrm{pp}$.

Cabrera, E. A. 1999. La gestión de opcraciones modernas y de la producción (GOMP). In Liller, $11 \mathrm{pp}$.

CBD 2001. Review of the elTiciency allid eflicacy of existing Legal Instruments applicable 10 Invasive Alico Species. UNEP-ONI:-CBD. $31 \mathrm{pp}$.

Del Rio, M. L.; D. Pariona; J. H. Cúrdova y G. Salmón 2001 . Especies Exúticas Invasoras. Informe Nacional del Perú-l Reunión de Trabajo sobre Especies Exóticas Invasoras (Brasilia 2001). I ibro de Ponencias, Documento $\mathrm{N}^{\circ} 9.11 \mathrm{pp}$.

ENDBP 2001. Perú: Estrategia Nacional subre Diversidal Biologica. CONAM-CBDCONADIB :138 pp

Fernández, M. A.: B. Mingo: R. R. Bernabé y D. Torres 1998. (jAIA-3. Edit Vicens Vives (I3arcelona), $362 \mathrm{pp}$.

Gosz, J. R., R. T. Holmes. G. E. Likens \& F. H. Bormann 1978. El flujo de energia en un sisterna de bosque. Investigación y Ciencia. 20:47-57.

Margaleff, R. 1974. Licologia. Ediciones OMEGA (Barcclona), $953 \mathrm{pp}$.

Odum, E. P. 1972. Ecologia. Editorial Inierumericana (México), $639 \mathrm{pp}$

Ojasti, J. 2001. Especics Exóticas Invasoras. Documento para la Estrategia Regional de la ('umunidad Andina de Naciones (CAN) ( $\mathrm{Cu}$ racas), $63 \mathrm{pp}$

Prescutt. J.: B. Gauthier y I. N. Mbongu 2000. Guia para deserrollar una Estrategia de Biodiversidad desfe una perspectiva de Desarrollo Sosienible. PNUMA (Montreal). $70 \mathrm{pp}$. 
Räuz-Luna. E. I: 2001. Impacto de la caza de subsistencia en la keserva de bióslera del Manu (Madre del Pios. Per(i) : Lecciones para la Gestión de Concrsación, 141-149.1.11:11 Vanu yotras experiencias de Investigación y Manejo de Bosques Ncotropicales (Rodrígucy. L. O. ed.), UNESCO: $308 \mathrm{pp}$.

Ricklet, R. E. 1998. The Economy of Nature. Editorial Médica Panamericana (Bs. As.), 692 pp.

YCEA 1994. Eicologiay Desarrollo Sustentable. Yachay Centro de Estudios y Asesoria (Lima), 238 pp.

\section{ANEXO}

Propuesta de Política Nacional sobre Desarrollo Sostenible en el corto plazo como marco para el Plan de Emergencia para los próximos cuatro años

Dido que no se desea poner obstáculos a los esfuerzos que se hacen por mejorar el estado actual del país, y aun cuando algunos temas no han sido tratados en el presente trabajo, se cree pertinentc incluir aquí una propuesla resumida que rebase el ámbito de la biodiversidad - no obstante saberse que el aporte de nuestra diversidad biológica y segúı los rubros está entre el $65 \%$ y el $95 \%$ a la economia nacional (ENDBP 2001)- a fin de vincularla a un conjunto de evidentes necesidades y aspiraciones nacionales. Los objetivos estratégicos y las acciones prioritarias que se dan a continuación tienen ese sentido.

\section{Objetivos Estratégicos:}

- Fomentar el desarrollo económico del país basándose en la utilización sostenible de la diversidad biológica, promoviendo la participacion del sector privado que comprende a las micro, pequeñas y medianas empresas, tanto a las formalmente establecidas como especialmente a las informales.

- Conservar la diversidad ecosistémica, especifica y genética del territorio peruano, así como los procesos ecológicos esenciales de los cuales depende la su pervivencia de las especies que lo habitan.
- Promover la parlicipación justa y equilativa en los beneficios que se deriven del uso de la divessidad biológica.

- Incentivar la educación. el desurrollo de capacidades liumanas e institucionales y el intercambio de información.

- Impulsar decididamente la imvestigación cientifica básica como eje fumdamental del Desarrollo. Ella es la que generará la creación y/o transferencia de tecnologias propias o adecuadas, y cvitará esa novedosa y peligrosa forma de dependencia que crean las tecnologías (y biotecnologias) importadas.

- Fomentar alianzas estratégicas y proactivas, incluso del sector privado consecuentes con el desarrollo sostenible.

\section{Acciones prioritarias}

- Un primer frente de acción debe estar orientado a mejorar la educación en todos sus niveles, empezando por una seria revisión y corrección de las leyes existenles en la materia, con el objetivo de que nusstros docentos (de colegios y universidades nacionales) rengan remuneraciones dignas y obligatoriedad de actualización-perfeccionamiento a fin de que estén en la mejor de las condiciones para la transferencia de sus conocimientos hacia nuestros hijos.

_-El segundo firente debe estar dirigido a:

a) atacar frontalmente a la corrupción mediante denuncias luego de investigaciones cuidadosas, y haciendo el seguimiento respectivo para que la Ley caiga con todo su peso sobre los corruptos; y

b) estudiar las leyes existentes para saber si ellas son herramientas eficientes para combatir la currupción, caso contrario proponer las enmiendas correspondientes y - por qué noformular nuevas. 
- El tercer frente lo debe constiturir el es tudio de la legislación sobre nuestros recursos naturales y sobre el fomento a la micro, pequeña y mediana empresa a fin de apreciar si son funciunales y compatibles con el devarrollo sostenible y-sobre todo si ticnen todos los componentes que permitan aliviar la economia de los sectores más deprimidos del pais. Esto traería como consecuencia la disminución de las tensiones y "tentaciones" ocasionadas por la incapacidad para solventar los gastos mínimos de las necesidades básicas de las familias más necesitadas, como son la alimentación. la vestimenta y la educación, que por otra parte son lambićn darcelos constitucionales que les asisten. Si la legislación no apunta hacia tal misión (dismimlicioin de tensiones sociales). habria que llegar hasta la propuesta de nuevas leyes para que la alcancen, y será crucial que la legislación del Perú estci orientada a hacer posible el desarrollo sostuible. En este punto, se debe enfatizar que el sectur informal habrá de ser estudiado y probablemente incentivado de manera especial dentro de su contexto, de tal suerte que pueda ser incorporado como el capital que los cstudiosos indican. Parece que solo asi se podrá construir al verdadero ntotor de la reactivación económica que precisa el pais $y$, probablemente, desarrollar los principales scctores generadores de puestus de trabajo. 